\title{
Emulation and Approximation of a Flexible Delay Line by Parallel Non-overtaking Delay Lines
}

\author{
Duan-Shin Lee, Kai-Jie Hsu, Cheng-Shang Chang, Jay Cheng \\ Institute of Communications Engineering \\ National Tsing Hua University \\ Hsinchu 300, Taiwan, R.O.C. \\ Email: lds@cs.nthu.edu.tw \\ \{cschang, jcheng\}@ee.nthu.edu.tw \\ g9562645@oz.nthu.edu.tw
}

\begin{abstract}
In this paper we propose to construct an flexible delay line with maximum delay $d$ by parallel non-overtaking delay lines. We show that for a fixed number of non-overtaking delay lines, an optimal policy to minimize packet losses is to assign arriving packets to the non-overtaking delay line that has the largest residual service time while maintaining the FIFO order for each non-overtaking delay lines. Based on this optimal policy we show that to exactly emulate an flexible delay line, one needs $[(d+1) / 2\rceil$ non-overtaking delay lines. We also show that if one can tolerate a small packet loss probability, one just needs $O(\sqrt{d})$ non-overtaking delay lines. In this case, we show that the residual service times of the non-overtaking delay lines behaved as if they followed the order statistics of uniform random variables.
\end{abstract}

Index Terms - optical buffer, flexible delay line, non-overtaking delay line, optimal policy, order statistics

\section{INTRODUCTION}

A most fundamental research issue in optical networks is to avoid optical-electric-optical conversion and to achieve alloptical packet switched networks. The most crucial device in an all-optical packet switched network is an optical buffer. Optical buffers are typically constructed using optical switches and fiber delay lines. Within an optical buffer, packets must be routed in a clever way to avoid conflicts and to achieve some certain constraints for the inputs and outputs of the optical buffer. In addition, since lasers and optical elements are expensive, one major design consideration of optical buffers is to reduce the construction complexity.

There is a surge of research results on optical queues recently. We refer the reader to [1], [2], [3], [4], [5], [6], [7] and the references cited therein. A discrete-time queue with a single input and a single output can be viewed as a switch that maps the time sequence of arrivals to the time sequence of departures. There is a correspondence between classic switches and optical queues [5]. Let $\tau^{a}\left(p_{n}\right)$ and $\tau^{d}\left(p_{n}\right)$ be the arrival time and the departure time of the $n^{\text {th }}$ packet respectively. A network element with a single input and a single output is called a flexible delay line (FDL) with maximum delay $d$ if it realizes the set of sample paths that satisfy

$$
\tau^{a}\left(p_{n}\right) \leq \tau^{d}\left(p_{n}\right) \leq \tau^{a}\left(p_{n}\right)+d, \quad \text { for all } n,
$$

and

$$
\tau^{d}\left(p_{m}\right) \neq \tau^{d}\left(p_{n}\right), \quad \text { for all } m \neq n .
$$

A network element with a single input and a single output is called a non-overtaking delay line (NDL) with maximum delay $d$ if it realizes the set of sample paths that satisfy (1) and

$$
\tau^{d}\left(p_{n}\right)<\tau^{d}\left(p_{n+1}\right)
$$

for all $n$. In queueing terminology, a flexible delay line is an infinite-server queue and a non-overtaking delay line is a firstin-first-out (FIFO) queue with known departure times. Among the classical switches, a flexible delay line corresponds to a strict sense nonblocking switch. A non-overtaking delay line corresponds to a Unimodal-Unimodal nonblocking switch [8].

The best known construction of an FDL with maximum delay $d$ is by Cantor networks. The construction complexity is $O\left((\log d)^{2}\right) 2 \times 2$ optical switches [5]. However, the routing of packets in this construction is very complicated. On the other hand, the construction of NDLs is simple and efficient. The construction complexity is only $O(\log d) 2 \times 2$ optical switches. In addition, the routing of packets through the construction is very simple. Packets are self-routed through the construction. In this paper we propose to construct an FDL with maximum delay $d$ by parallel NDLs (Figure 1). We show that for a fixed number of NDLs $B$, an optimal policy to minimize packet losses is to assign arriving packets to the NDL that has the largest residual service time while maintaining the FIFO order for each NDL. Based on this optimal policy we show that to exactly emulate an FDL, one needs $\lceil(d+1) / 2\rceil$ NDLs. We also show that if one can tolerate a small packet loss probability, one just needs $O(\sqrt{d})$ NDLs. In the latter case, we show that the residual service times of the non-overtaking delay lines behaved as if they followed the order statistics of uniform random variables. In either case, the routing of packets is very simple.

This paper is organized as follows. In Section II we show that the policy that selects the NDL with largest residual service time while maintaining the FIFO order for all NDLs is optimal in minimizing the number of lost packets. In Section 
III we show that one needs $\lceil(d+1) / 2\rceil$ NDLs to exactly emulate an FDL. In Section IV we show that one just needs $O(\sqrt{d})$ NDLs if a small packet loss probability is allowed. Finally, the conclusion of this paper is given in Section VI.

\section{Optimal Policy}

In this paper we consider a system of $B$ parallel nonovertaking delay lines (NDLs) that emulate or approximate a flexible delay line (FDL). Please see Figure 1 for a diagrammatic illustration. Each non-overtaking delay line and the flexible delay line allow maximum delay $d$. Specifically, new packets arrive at the parallel NDLs. Each packet requests a delay in the range from zero to $d$. There is at most one packet arrival in each time slot and packets request delay in such a way that at most one packet departs from the system in each time slot. We now give an example to review the sample paths of a flexible delay line. The length of packets is fixed and is equal to one time slot. Part (a) of Figure 2 shows a sample path of an FDL with maximum delay $d=5$ time slots. The first three packets request delays of 5, 3 and 5 times slots respectively, and they will depart the flexible delay line in time slots 5, 6 and 8 respectively. If the packet arriving at time 4 requests a delay of 1,2 or 4 , it will be dropped as there are already packets scheduled to depart in these time slots (see Eq. (2)). On the other hand, if the requested delay of the fourth packet is 0,3 or 5 , the flexible delay line will accept it. Part (b) of Figure 2 shows $B=3$ non-overtaking delay lines. Packets enter the NDLs from the left side and depart from the right side. The position of the packets represents the number of time slots that they need to exit the NDLs at time $t=4$. At time $t=4$, if the requested delay of the fourth packet is 3 or 5 , the fourth packet can be accepted by the second or the third NDLs without overtaking existing packets in the NDLs. However, if the requested delay is zero, the fourth packet cannot be accepted due to the lack of idle NDLs. However, if the first three packets were scheduled so that their positions in the NDLs are as shown in part (c) of the figure, the fourth packet can be accepted even if its requested delay is zero. From this example, it is clear that the number of NDLs needed to emulate exactly an FDL depends on the packet assignment policy.

In this section we study the policy of assigning new packets to NDLs such that later packets do not overtake earlier packets and packet loss is minimized. We begin by describing the dynamics of a flexible delay line with maximum delay $d$. Let $s(t)$ be a $1 \times d$ vector and its $i^{t h}$ entry $s_{i}(t)$ indicates whether a packet is scheduled to depart at time $t+i$, where $i=0,1, \ldots, d-1$. Specifically, $s_{i}(t)=1$ if there is a packet scheduled to depart at time $t+i$, and $s_{i}(t)=0$ otherwise. Let the requested delay of the packet arriving at time $t$ be denoted by $d(t)$. Recall that an important constraint of a flexible delay line is that a flexible delay line allows at most one packet to depart in each time slot. Therefore, $d(t) \neq i$, if $s_{i}(t)=1$. Then we have the following recursive equation

$$
s_{i}(t+1)= \begin{cases}s_{i+1}(t), & \text { if } d(t) \neq i+1 \\ 1, & \text { if } d(t)=i+1\end{cases}
$$

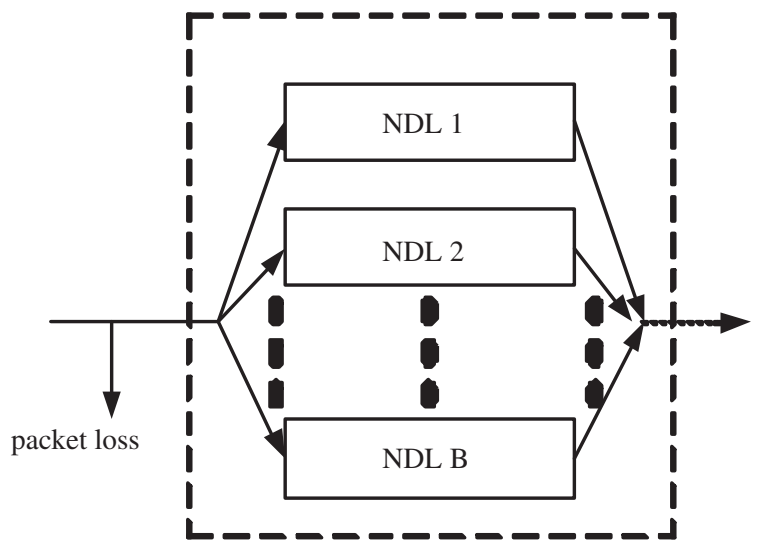

Fig. 1. A flexible delay line with maximum delay $d$ constructed by $B$ parallel non-overtaking delay lines

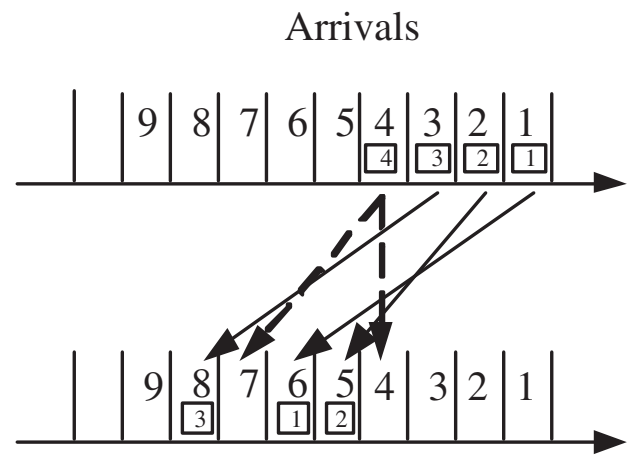

Departures

(a) A sample path of a flexible delay line

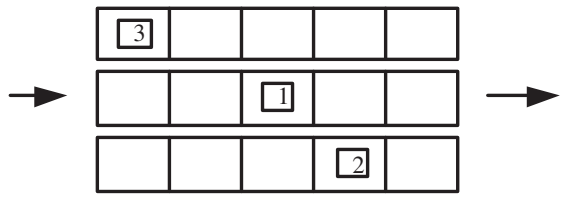

(b) Three non-overtaking delay lines with progressing packets

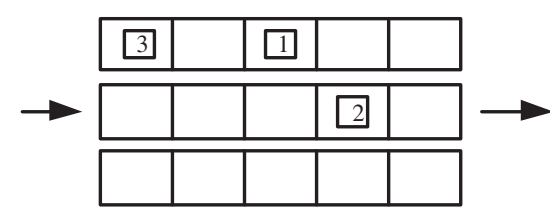

(c) A different assignment policy of packets

Fig. 2. An example to illustrate the sample path of a flexible delay line with maximum delay five time slots and packets progressing in three parallel non-overtaking delay lines 
for $i=0,1, \ldots, d-2$. In addition, $s_{d-1}(t+1)=0$ if $d(t) \neq d$. Non-overtaking delay lines have a similar dynamics. Consider the $i^{t h}$ NDL in Figure 1 and let $\boldsymbol{v}_{i}(t)$ be a $1 \times d$ vector. Let $v_{i, j}(t)$ be the $j^{t h}$ entry of $\boldsymbol{v}_{i}(t)$. if there is a packet scheduled to depart from the NDL at time $t+j$, then $v_{i, j}(t)=1$. Otherwise, $v_{i, j}(t)=0$. Define

$$
r_{i}(t) \stackrel{\text { def }}{=} \max \left\{j \mid v_{i, j}(t)=1, j=0,1, \ldots, d-1\right\} .
$$

If $v_{i, j}(t)=0$ for all $j, r_{i}(t)$ is defined to be zero. We call $r_{i}(t)$ the residual service time of the $i^{\text {th }}$ NDL at time $t$. The residual service time of an NDL at time $t$ is the time interval from now to the instant when the NDL becomes idle if no future arrivals to this NDL occur. Let $\boldsymbol{r}(t)$ be a $1 \times B$ vector where the $i^{\text {th }}$ entry is $r_{i}(t)$. An arriving packet with requested delay $d(t)$ can only be admitted into the $i^{t h}$ NDL if $d(t)>r_{i}(t)$ in order not to violate constraint (3). Therefore, if a new arriving packet is admitted into the $i^{t h} \mathrm{NDL}$, the dynamics of the $i^{\text {th }} \mathrm{NDL}$ is as follows

$$
v_{i, j}(t+1)= \begin{cases}v_{i, j+1}(t), & \text { if } d(t) \neq j+1 \\ 1, & \text { if } d(t)=j+1>r_{i}(t) .\end{cases}
$$

Let

$$
\begin{aligned}
F_{+} & =\left\{i \mid d(t)>r_{i}(t)>0\right\} \\
F_{0} & =\left\{i \mid r_{i}(t)=0\right\}
\end{aligned}
$$

and

$$
F=F_{+} \cup F_{0}
$$

Any NDL in set $F$ is said to be fit, as it can accept the new arrival without violating the non-overtaking constraint.

We now consider a packet assignment policy for the $B$ parallel NDLs in Figure 1. This policy assigns a new arriving packet to the NDL that is in $F$ and has the largest residual service time. Denote this policy by $\pi^{\star}$. Given the vector of the residual service times, $\boldsymbol{r}(t)$, policy $\pi^{\star}$ selects the NDL that has the largest residual service time, i.e.

$$
\pi^{\star}(\boldsymbol{r}(t), d(t))=\operatorname{argmax}\left\{r_{i}(t) \mid i \in F_{+}\right\}
$$

if $F_{+}$is nonempty. On the other hand if $F_{+}$is empty and $F_{0}$ is nonempty, then policy $\pi^{\star}$ selects the first idle NDL, i.e.

$$
\pi^{\star}(\boldsymbol{r}(t), d(t))=\min F_{0} .
$$

Otherwise, the new arrival is dropped due to lack of NDLs. Note that if set $F$ is nonempty, policy $\pi^{\star}$ will not drop the new arrival. We say that a policy is a best effort policy if the policy drops a new arrival only when there is no NDL that can accept the new arrival without violating Eq. (3). Policy $\pi^{\star}$ is a best effort policy. Note that (7) can be rewritten as

$$
\pi^{\star}(\boldsymbol{r}(t), d(t))=\operatorname{argmin}\left\{d(t)-r_{i}(t) \mid i \in F_{+}\right\} .
$$

Thus, policy $\pi^{\star}$ assigns a new arrival to the NDL whose residual service time $r_{i}(t)$ is nearest to the delay request $d(t)$ among all NDLs in $F_{+}$. Since the NDLs in $F_{+}$are said to be fit, thus we can say that policy $\pi^{\star}$ is a best-fit policy. In fact, later in this section we will show that policy $\pi^{\star}$ minimizes the accumulated number of dropped packets.
A sample path of an FDL is the collection of arrival and departure instants, i.e. $\left\{\left(\tau_{a}\left(p_{n}\right), \tau^{d}\left(p_{n}\right)\right) \mid n=1,2, \ldots\right\}$. Alternatively, we say that arrival and departure instants are generated by the sample paths. We say that a sample path is feasible if Eqs. (1) and (2) hold for all packets in this sample path. In a feasible sample path, the requested delays of all arrivals are such that no two packets compete for a common time slot for departure. Thus, there is no packet loss by the FDL in a feasible sample path. In the rest of this paper, whenever we mention feasible sample paths, we mean the feasible sample path of the FDL being emulated or approximated by the NDLs in Figure 1.

In the remainder of this section, we will show that policy $\pi^{\star}$ is an optimal policy among all the best effort policies if the arriving packets are generated by a feasible sample path of the FDL being emulated or approximated by the NDLs in Figure 1. Let $\ell_{\pi}(\boldsymbol{r}(t), t)$ be the number of lost packets at time $t$, if policy $\pi$ is used and the vector of residual service times is $\boldsymbol{r}(t)$ at time $t$. Let $L_{\pi}\left(\boldsymbol{r}\left(t_{1}\right), t_{1}, t_{2}\right)$ be the accumulated number of lost packets in interval $\left[t_{1}, t_{2}\right]$, i.e.

$$
L_{\pi}\left(\boldsymbol{r}\left(t_{1}\right), t_{1}, t_{2}\right)=\sum_{t=t_{1}}^{t_{2}} \ell_{\pi}(\boldsymbol{r}(t), t)
$$

We remark that in the rest of this paper we are interested only in feasible sample paths. Since the arrival and departure instants are generated by a feasible sample path, packets are dropped by the parallel NDLs only due to lack of NDLs. Thus, in our analysis, we do not need to keep the detail state information that is described in (4) and (6). One just needs to keep track of the residual service times of the NDLs.

The following theorem is the main result of this section. Its proof is given in Appendix A.

Theorem 1 If $\left\{\left(\tau^{a}\left(p_{n}\right), \tau^{d}\left(p_{n}\right)\right) \mid n \geq 1\right\}$ is a feasible sample path to an FDL with maximum delay $d$, then

$$
L_{\pi^{\star}}\left(\boldsymbol{r}\left(t_{1}\right), t_{1}, t_{2}\right) \leq L_{\pi}\left(\boldsymbol{r}\left(t_{1}\right), t_{1}, t_{2}\right)
$$

for any best effort policy $\pi, 0 \leq t_{1} \leq t_{2}$ and any initial residual service time vector $\boldsymbol{r}\left(t_{1}\right)$.

\section{EXACT EMULATION}

The main objective of this section is to study the number of NDLs that are needed to emulate an FDL with maximum delay $d$ exactly. The main result is stated in the following theorem. Its proof is given in Appendix B.

Theorem 2 A system of B parallel non-overtaking delay lines with $B \geq\lceil(d+1) / 2\rceil$ can emulate exactly a flexible delay line with maximum delay $d$, if the non-overtaking delay lines adopt policy $\pi^{\star}$ and start from an empty system.

We remark that Theorem 2 holds if the arrival and departure instants are generated by an infeasible sample path. We remark that since policy $\pi^{\star}$ minimizes the number of lost packets for any feasible sample path, it needs the least number of NDLs to avoid any packet loss among all best effort policies. Thus, 
$\lceil(d+1) / 2\rceil$ is the minimum number of NDLs in order to emulate an FDL with maximum delay $d$.

\section{APPROXIMATION}

In the last section we show that to emulate exactly a flexible delay line with maximum delay $d$, one needs $\lceil(d+1) / 2\rceil$ nonovertaking delay lines. In practice, one may not need to exactly emulate a flexible delay line. If one uses less than $\lceil(d+1) / 2\rceil$ NDLs, packets can be lost due to insufficient NDLs. One may be able to tolerate a small packet loss probability and uses a smaller number of non-overtaking delay lines. In Figure 3 we show the number of NDLs needed in order to achieve $10^{-5}$ packet loss probability assuming that the requested delays of arriving packets are uniformly distributed and independent with everything else. In this simulation, packets are assigned to the NDLs according to policy $\pi^{\star}$. The length of simulation time is one million time slots. From Figure 3, we see that one only needs $O(\sqrt{d})$ NDLs to guarantee a small packet loss probability. In Figure 4, we show the expected residual service time as a function of the NDL indexes. From Figure 4, to approximate an FDL with maximum delay 2000 (resp. 500) time slots, one needs about 35 (resp. 17) NDLs. The expected residual service times of further NDLs are all nearly zero. More importantly, note that the expected residual service times are linearly decreasing from the corresponding maximum delay to zero. This implies that the residual service times are roughly uniformly distributed. Particularly, the residual service times when policy $\pi^{\star}$ is used are always arranged in a descending order according to Corollary 6 . Thus, the residual service time of the $i^{\text {th }} \mathrm{NDL}$ is the $i^{\text {th }}$ largest uniform random variable in the range $[0, d]$. In this section, we shall give an intuitive derivation of this phenomena.

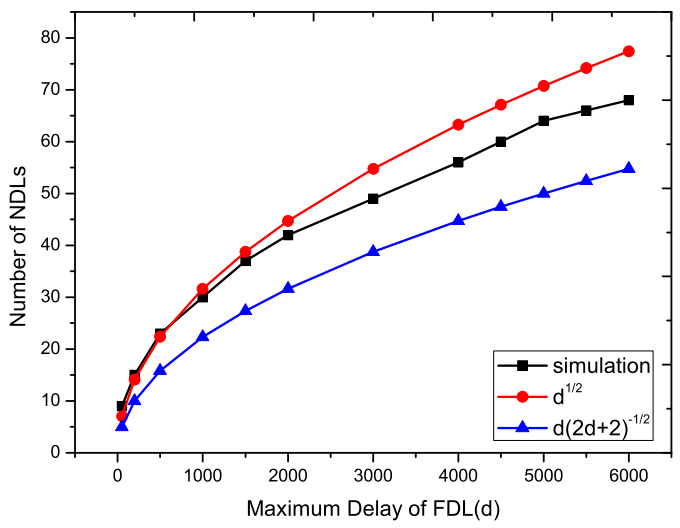

Fig. 3. The number of non-overtaking delay lines needed to approximate a flexible delay line with maximum delay $d$

If we assume that the requested delays of packets are generated by independent random variables, then packets can be dropped by the parallel NDLs in Figure 1 for two reasons. First, packets can be dropped due to violation of the single arrival and departure per time slot constraint. Second, a new

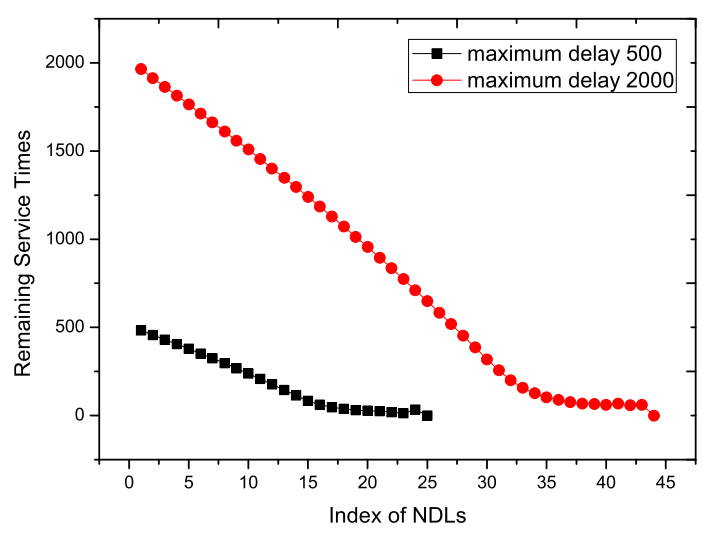

Fig. 4. The expected residual service times of 34 parallel non-overtaking delay lines with maximum delay 2000 and 500

arriving packet can be dropped if it overtakes all existing packets in the NDLs and no idle NDLs are available to accept it. An exact analysis of the system in Figure 1 with policy $\pi^{\star}$ is very complicated. One needs to include the residual service times of all existing packets in the state description. This makes it a very complicated Markov chain. In this section we ignore the constraint on single arrival and departure per time slot. Let $X_{i}(t)$ be the residual service time of the $i^{t h}$ NDL at time $t$. According to Corollary $6, X_{i}(t)>X_{i+1}(t)$ for all $t$. Let $Y_{i}(t)=X_{i-1}(t)-X_{i}(t)$ for $i=2,3, \ldots, B$ and $Y_{1}(t)=d+1-X_{1}(t)$. We approximate the residual service times of the NDLs by the model described as follows. Consider an interval between $A$ and $B$ that has length $d+1$ as shown in Figure 5. Denote this interval by $\overline{A B}$. Segment $\overline{A B}$ into consecutive subintervals of lengths $Y_{1}(t), Y_{2}(t)$ and so on. The values of $X_{1}(t), X_{2}(t), \ldots$, are also shown on the figure as well. In each time slot, two events occur to the model. First, an integer-valued random variable $d(t)$ uniformly distributed in $[0, d]$ is drawn and placed on $\overline{A B}$. Suppose that as shown in Figure $5 d(t)$ falls in the interval $Y_{i}(t)$. The right end point of the interval $Y_{i}(t)$ is moved to the point where $d(t)$ falls and the thick interval designates the new value for $Y_{i}(t+1)$. This event corresponds to the arrival of a new packet. In the second type of events, the end points of the intervals $Y_{1}(t), Y_{2}(t)$ and so on, are moved to the right by one unit. This event corresponds to the time advancement in Definition 2. As a result, the length of the first interval $Y_{1}(t)$ is incremented by one slot, that of the last subinterval near point $B$ is decremented by one and those of the rest intervals are unchanged.

We now derive a dynamic equation for the process $Y_{i}(t)$. Consider three disjoint events: the delay request $d(t)$ of a new arriving packet falls in interval $Y_{i}(t), d(t)$ falls in interval $Y_{i-1}(t)$ and that $d(t)$ does not fall in $Y_{i}(t)$ nor in $Y_{i-1}(t)$. From Figure 5 it is obvious that these three cases correspond equivalently to that $d(t)$ is in the interval $\left(X_{i}(t), X_{i-1}(t)\right)$, $d(t)$ is in the interval $\left(X_{i-1}(t), X_{i-2}(t)\right)$, and $d(t)$ is in the union $\left[0, X_{i}(t)\right) \cup\left(X_{i-2}(t), d+1\right]$, respectively. Thus, based 
on decomposing the total probability space according to these three disjoint events, we have

$$
\begin{aligned}
Y_{i}(t+1)= & Y_{i}(t+1) I_{\left\{X_{i}(t)<d(t)<X_{i-1}(t)\right\}}+ \\
& Y_{i}(t+1) I_{\left\{X_{i-1}(t)<d(t)<X_{i-2}(t)\right\}}+ \\
& Y_{i}(t+1) I_{\left\{d(t)<X_{i}(T) \text { or } d(t)>X_{i-2}(t)\right\}}
\end{aligned}
$$

The first term in (11) corresponds to the case where $d(t)$ falls in interval $Y_{i}(t)$ (see Figure 5). In this case $Y_{i}(t+1)$ is the thick interval in Figure 5 and clearly

$$
Y_{i}(t+1) I_{\left\{X_{i}(t)<d(t)<X_{i-1}(t)\right\}}=X_{i-1}(t)-d(t) .
$$

The second term in (11) corresponds to the case where $d(t)$ falls in interval $Y_{i-1}(t)$. In this case,

$$
\begin{aligned}
& Y_{i}(t+1) I_{\left\{X_{i-1}(t)<d(t)<X_{i-2}(t)\right\}} \\
= & Y_{i}(t)+\left[Y_{i-1}(t)-\left(X_{i-2}(t)-d(t)\right)\right] \\
= & d(t)-X_{i}(t) .
\end{aligned}
$$

The third term in (11) corresponds to the case where $d(t)$ does not fall in $Y_{i}(t)$ nor in $Y_{i-1}(t)$. In this case, as time is advanced by one time slot, all interval end points on $\overline{A B}$ are moved to the right by one slot, $Y_{i}(t+1)$ equals to $Y_{i}(t)$. Specifically,

$$
Y_{i}(t+1) I_{\left\{d(t)<X_{i}(T) \text { or } d(t)>X_{i-2}(t)\right\}}=Y_{i}(t) .
$$

Substituting (12), (13) and (14) into (11), we obtain

$$
\begin{aligned}
& Y_{i}(t+1)= \\
& \quad\left(X_{i-1}(t)-d(t)\right) I_{\left\{X_{i}(t)<d(t)<X_{i-1}(t)\right\}}+ \\
& \quad\left(d(t)-X_{i}(t)\right) I_{\left\{X_{i-1}(t)<d(t)<X_{i-2}(t)\right\}}+ \\
& Y_{i}(t) I_{\left\{d(t)<X_{i}(T) \text { or } d(t)>X_{i-2}(t)\right\}} .
\end{aligned}
$$

If the $i^{t h} \mathrm{NDL}$ is idle at time $t, Y_{i}(t)$ is the right most subinterval on $\overline{A B}$ and the last term on the right hand side of (15) is replaced by

$$
\left(Y_{i}(t)-1\right)^{+} I_{\left\{d(t)>X_{i-2}(t)\right\}},
$$

where $(x)^{+}=\max (x, 0)$. Similarly, one can derive the following recursive equation for $Y_{1}(t)$

$$
\begin{aligned}
Y_{1}(t+1)= & \left(Y_{1}(t)+1\right) I_{\left\{d(t)<X_{1}(t)\right\}} \\
& +(d+1-d(t)) I_{\left\{d(t)>X_{1}(t)\right\}} .
\end{aligned}
$$

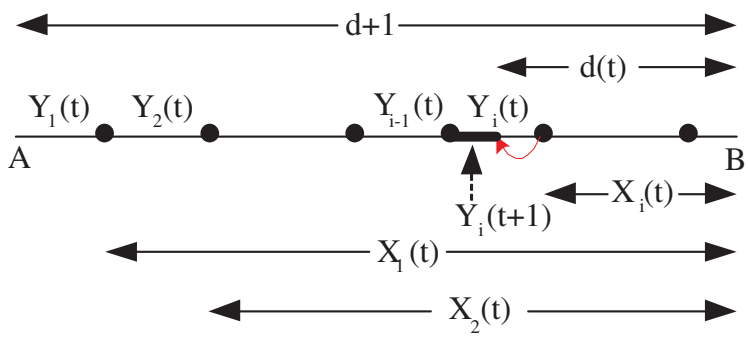

Fig. 5. Dynamics of the $Y_{i}(t)$ intervals
We are interested in $y_{i} \stackrel{\text { def }}{=} \lim _{t \rightarrow \infty} \mathrm{E}\left[Y_{i}(t)\right]$. To this end, (15) and (16) are still too complicated. We now derive an approximate recursive equation for $y_{i}$. We assume for the time being that $y_{i+1}$ is not the last interval on $\overline{A B}$. Again consider three disjoint cases: $d(t)$ is in the interval $\left(X_{i}(t), X_{i-1}(t)\right)$, $d(t)$ is in the interval $\left(X_{i-1}(t), X_{i-2}(t)\right)$, and $d(t)$ is in the union $\left[0, X_{i}(t)\right) \cup\left(X_{i-2}(t), d+1\right]$. From the definition of conditional expectations, we have

$$
\begin{aligned}
& \mathrm{E}\left[Y_{i}(t+1)\right]= \\
& \mathrm{E}\left[Y_{i}(t+1) \mid X_{i}(t)<d(t)<X_{i-1}(t)\right] \\
& \quad \times \operatorname{Pr}\left[X_{i}(t)<d(t)<X_{i-1}(t)\right]+ \\
& \mathrm{E}\left[Y_{i}(t+1) \mid X_{i-1}(t)<d(t)<X_{i-2}(t)\right] \\
& \quad \times \operatorname{Pr}\left[X_{i-1}(t)<d(t)<X_{i-2}(t)\right]+ \\
& \mathrm{E}\left[Y_{i}(t+1) \mid d(t)<X_{i}(T) \text { or } d(t)>X_{i-2}(t)\right] \\
& \quad \times \operatorname{Pr}\left[d(t)<X_{i}(T) \text { or } d(t)>X_{i-2}(t)\right]
\end{aligned}
$$

To obtain an expression for the conditional expectations and the probabilities of conditions in (17), we approximate and assume that $Y_{i}(t)=y_{i}$ for all $i$. Now consider the two terms in the first product on the right hand side of (17). We approximate the two terms by

$$
\begin{aligned}
& \mathrm{E}\left[Y_{i}(t+1) \mid X_{i}(t)<d(t)<X_{i-1}(t)\right] \approx \frac{y_{i}}{2} \\
& \operatorname{Pr}\left[X_{i}(t)<d(t)<X_{i-1}(t)\right] \approx \frac{y_{i}}{d+1} .
\end{aligned}
$$

To see the motivation for (18), note that the condition in (18) is equivalent to that $d(t)$ falls within the interval $Y_{i}(t)$. According to the earlier discussion, the new value $Y_{i}(t+1)$ is one half of the old value $Y_{i}(t)$. Note that we assume that $Y_{i}(t)=$ $y_{i}$. Thus, we obtain (18). To obtain an approximation for the probability in (19), we note that $d(t)$ is uniformly distributed over $[0, d]$. Thus, one has (19). Based on the same argument, we approximate the expectation of $Y_{i}(t+1)$ conditioned on the event that $d(t)$ falls in interval $Y_{i-1}(t)$. Specifically,

$$
\begin{aligned}
& \mathrm{E}\left[Y_{i}(t+1) \mid X_{i-1}(t)<d(t)<X_{i-2}(t)\right] \\
& \quad \approx \frac{y_{i-1}}{2}+y_{i} \\
& \operatorname{Pr}\left[X_{i-1}(t)<d(t)<X_{i-2}(t)\right] \approx \frac{y_{i-1}}{d+1} .
\end{aligned}
$$

Finally, we approximate the expectation of $Y_{i}(t+1)$ conditioned on the event that $d(t)$ does not fall in interval $Y_{i}(t)$ nor in interval $Y_{i-1}(t)$ by

$$
\mathrm{E}\left[Y_{i}(t+1) \mid d(t)<X_{i}(T) \text { or } d(t)>X_{i-2}(t)\right] \approx y_{i} .
$$

We approximate the probability of the condition by

$$
\operatorname{Pr}\left[d(t)<X_{i}(T) \text { or } d(t)>X_{i-2}(t)\right] \approx 1-\frac{y_{i-1}+y_{i}}{d+1} .
$$

We substitute Eqs. (18-23) to (17) and invoke the stationarity assumption on $y_{i}$ to obtain

$$
y_{i}=\frac{y_{i}}{2} \frac{y_{i}}{d+1}+\left(\frac{y_{i-1}}{2}+y_{i}\right) \frac{y_{i-1}}{d+1}+y_{i}\left(1-\frac{y_{i-1}+y_{i}}{d+1}\right) .
$$


With the same argument we can show that if $y_{i}$ is the last interval on $\overline{A B}$, then (24) is modified as

$$
\begin{aligned}
y_{i}=( & \left.\frac{y_{i-1}}{2}+y_{i}-1\right)^{+} \frac{y_{i-1}}{d+1}+\left(\frac{y_{i}}{2}-1\right)^{+} \frac{y_{i}}{d+1} \\
& +\left(y_{i}-1\right)^{+}\left(1-\frac{y_{i-1}+y_{i}}{d+1}\right) .
\end{aligned}
$$

Similarly, we derive the following equations for $y_{1}$ :

$$
y_{1}=\left(\frac{y_{1}}{2}+1\right) \frac{y_{1}}{d+1}+\left(1+y_{1}\right)\left(1-\frac{y_{1}}{d+1}\right) .
$$

From Eq. (26) we can solve $y_{1}=\sqrt{2(d+1)}$. Substituting this solution into Eq. (24), we obtain $y_{i}=y_{i-1}$ for all $i=$ $2,3, \ldots$, if $y_{i+1}>1$. However, if $y_{i+1}<1$, we substitute $y_{i-1}=\sqrt{2(d+1)}$ into (25). To solve (25), one considers cases in which $y_{i} \geq 2,1 \leq y_{i}<2$ and $0 \leq y_{i}<1$. Analysis of the above cases conclude that $y_{i}=1$. We mention that if $y_{i-1}=1$, the solution of (25) is $y_{i}=0$. With an unlimited number of NDLs available, assume that on average only $n$ NDLs are occupied. There are $n+1$ intervals. Since the total length of the intervals is $d+1$, we have

$$
\sqrt{2(d+1)} \times n+1=d+1,
$$

which gives $n=d / \sqrt{2(d+1)}$. The number of NDLs predicted by this formula is shown in Figure 3 for comparison.

\section{Simulation Studies}

In this section we present more simulation results. The setting and the simulation parameters have been described in Section IV. We show the expected residual service times of 43, 40, 30 and 20 NDLs with maximum delay 2000 in Figure 6. We can see that the expected residual service time decreases linearly from 2000 to zero for $B=43$ and $B=40$. In this case, there are sufficient NDLs. However, if there are insufficient NDLs, for instance $B=20$ or $B=30$, the expected residual service times still decreases linearly. However, it does not decrease to zero. We repeat this experiment for $d=500$ (shown in Figure 7) and observe the same phenomenon.

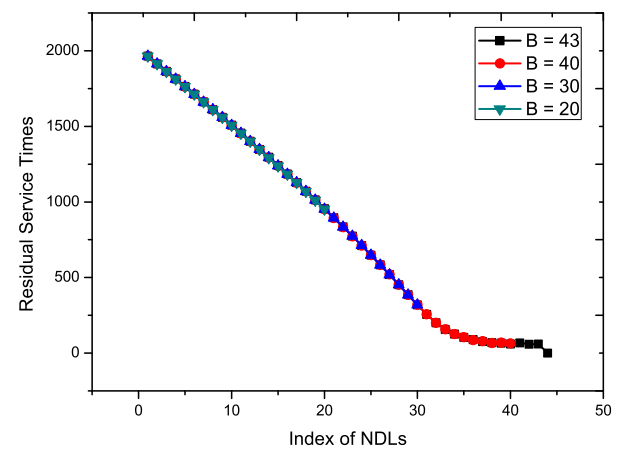

Fig. 6. The expected residual service times of various number of NDLs with maximum delay 2000

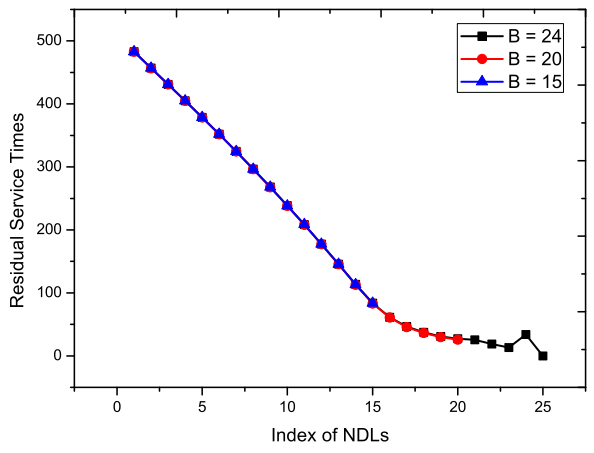

Fig. 7. The expected residual service times of various number of NDLs with maximum delay 500

Next we study the effect of delay distributions to the expected residual service times. Consider a normal distribution that has the same mean and variance as that of a random variable uniformly distributed on $[0, d]$. We then truncate and normalize the normal distribution to the range $[0, d]$ and use it to generate requested delays. In Figure 8 we show the expected residual service times of various numbers of NDLs. We see that although the delays are normally distributed, the residual service times are as if they follow the order statistics of uniform random variables. The expected residual service times decrease linearly. We then draw the expected residual service times for uniformly distributed requested delays versus normally distributed delays in Figure 9. From this figure, we conjecture that the residual service time is insensitive to the delay distributions. We draw the number of NDLs needed in order to achieve less than or equal to $10^{-5}$ loss probability in Figure 10 assuming that delay has a normal distribution. From this figure, we conjecture that the number of NDLs needed to approximate an FDL is insensitive to the delay distributions. Finally, we show the packet loss probability as a function of $B$ in Figure 11 for $d=2000$. From this figure we see that the packet loss probability is insensitive to the distribution of the requested delay. Moreover, the packet loss probability decreases very rapidly as $B$ is near $O(\sqrt{d})$.

\section{CONCLUSION}

In this paper we propose to construct an FDL with maximum delay $d$ by parallel NDLs (Figure 1). We show that for a fixed number of NDLs $B$, the optimal policy to minimize packet losses is to assign arriving packets to the NDL that has the largest residual service time while maintaining the FIFO order for each NDL. Based on this optimal policy we show that to exactly emulate an FDL, one needs $\lceil(d+1) / 2\rceil$ NDLs. We also show that if one can tolerate a small packet loss probability, one just needs $O(\sqrt{d})$ NDLs. In the latter case, we show that the residual service times of the non-overtaking delay lines behaved as if they followed the order statistics of uniform random variables.

For future work, we note that the mean value analysis 


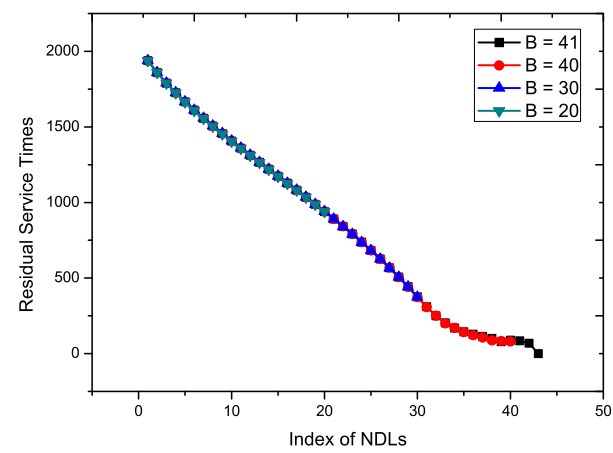

Fig. 8. The expected residual service times with normally distributed delay

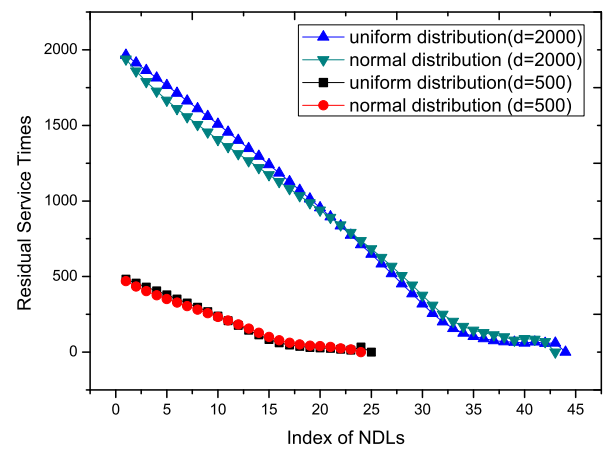

Fig. 9. Comparison of the expected residual service times for uniformly distributed delay and normal delay

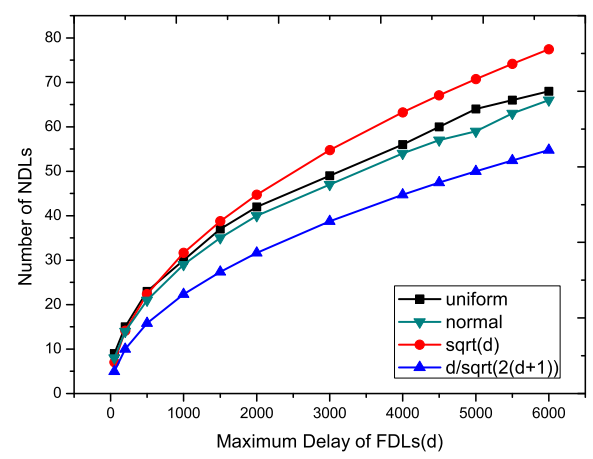

Fig. 10. The number of NDLs needed to approximate an FDL with maximum delay $d$

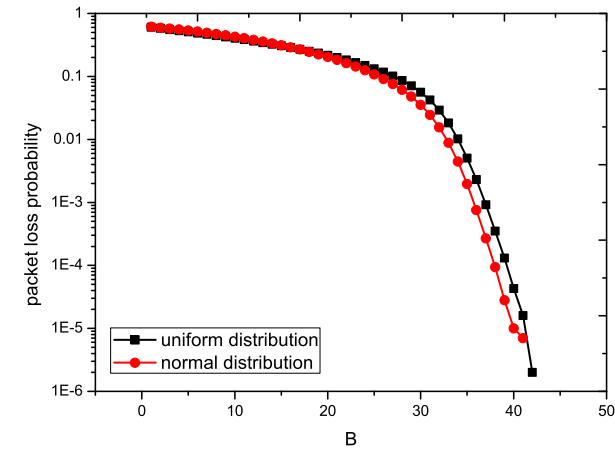

Fig. 11. The packet loss probability as a function of $B$

presented in Section IV can not explain why the expected residual service times decrease linearly for nonuniform requested delays. The analysis assumes that there is exactly one arriving packet in each time slot. For future works, it is interesting to study the residual service times for systems with non-uniformly distributed requested delays or less than $100 \%$ loaded packet arrivals.

\section{ACKNOWLEDGMENT}

This research is supported in part by the National Science Council, Taiwan, R.O.C., under Contract 95-2221-E-007-047MY3, and the Program for promoting Academic Excellence of Universities NSC 94-2752-E-007-002-PAE.

\section{APPENDIX A}

In this appendix we present the proof of Theorem 1. To prove Theorem 1 we need the following two lemmas. We have omitted the proof of Lemma 3. For Lemma 4 we note that $\boldsymbol{a} \geq \boldsymbol{b}$ if any entry of vector $\boldsymbol{a}$ is greater than or equal to the corresponding entry of vector $\boldsymbol{b}$.

Lemma 3 Let $\tilde{\boldsymbol{r}}$ be obtained from $\boldsymbol{r}$ by permuting the indexes of the NDLs. Then, for any history independent policy $\pi$ and any $t_{1} \leq t_{2}$ we have

$$
L_{\pi}\left(\boldsymbol{r}, t_{1}, t_{2}\right)=L_{\pi}\left(\tilde{\boldsymbol{r}}, t_{1}, t_{2}\right) .
$$

Lemma 4 If $\boldsymbol{r}^{\prime}\left(t_{1}\right) \geq \boldsymbol{r}\left(t_{1}\right)$, then

$$
L_{\pi^{\star}}\left(\boldsymbol{r}\left(t_{1}\right), t_{1}, t_{2}\right) \leq L_{\pi^{\star}}\left(\boldsymbol{r}^{\prime}\left(t_{1}\right), t_{1}, t_{2}\right)
$$

for any $t_{1} \leq t_{2}$ and for any feasible sample path.

Proof of Lemma 4. We prove this lemma by induction. If $t_{2}-t_{1}=0$,

$$
L_{\pi^{\star}}\left(\boldsymbol{r}\left(t_{1}\right), t_{1}, t_{1}\right)=\ell_{\pi^{\star}}\left(\boldsymbol{r}\left(t_{1}\right), t_{1}\right) .
$$

Since the arrival and departure instants are generated by a feasible sample path of the FDL being emulated or approximated, a new arriving packet can be lost only if $d\left(t_{1}\right)<r_{i}\left(t_{1}\right)$ for all $i$. Thus,

$$
\ell_{\pi^{\star}}\left(\boldsymbol{r}\left(t_{1}\right), t_{1}\right)=I_{\left\{\bigcap_{i=1}^{B}\left\{d\left(t_{1}\right)<r_{i}\left(t_{1}\right)\right\}\right\}}
$$


which implies that

$$
\ell_{\pi^{\star}}\left(\boldsymbol{r}\left(t_{1}\right), t_{1}\right) \leq \ell_{\pi^{\star}}\left(\boldsymbol{r}^{\prime}\left(t_{1}\right), t_{1}\right) .
$$

Now assume that (28) holds for any $t_{2}-t_{1}=T$ and for any feasible sample path. We need to show that (28) holds for any $t_{2}-t_{1}=T+1$ as well. From (9), it follows that

$$
\begin{aligned}
L_{\pi^{\star}}\left(\boldsymbol{r}\left(t_{1}\right), t_{1}, t_{2}\right)= & \ell_{\pi^{\star}}\left(\boldsymbol{r}\left(t_{1}\right), t_{1}\right)+ \\
& L_{\pi^{\star}}\left(\boldsymbol{r}\left(t_{1}+1\right), t_{1}+1, t_{2}\right)
\end{aligned}
$$

and

$$
\begin{aligned}
L_{\pi^{\star}}\left(\boldsymbol{r}^{\prime}\left(t_{1}\right), t_{1}, t_{2}\right)= & \ell_{\pi^{\star}}\left(\boldsymbol{r}^{\prime}\left(t_{1}\right), t_{1}\right)+ \\
& L_{\pi^{\star}}\left(\boldsymbol{r}^{\prime}\left(t_{1}+1\right), t_{1}+1, t_{2}\right) .
\end{aligned}
$$

Since $\boldsymbol{r}\left(t_{1}\right) \leq \boldsymbol{r}^{\prime}\left(t_{1}\right)$, from (7) and (8) it follows that

$$
k \stackrel{\text { def }}{=} \pi^{\star}\left(\boldsymbol{r}\left(t_{1}\right), d(t)\right) \leq k^{\prime} \stackrel{\text { def }}{=} \pi^{\star}\left(\boldsymbol{r}^{\prime}\left(t_{1}\right), d(t)\right) .
$$

Consider $\boldsymbol{r}\left(t_{1}+1\right)$ and $\boldsymbol{r}^{\prime}\left(t_{1}+1\right)$. For $i$ not equal to $k$ nor $k^{\prime}$, we have

$$
r_{i}\left(t_{1}+1\right)=\left(r_{i}\left(t_{1}\right)-1\right)^{+} \leq\left(r_{i}^{\prime}\left(t_{1}\right)-1\right)^{+}=r_{i}^{\prime}\left(t_{1}+1\right),
$$

where $(x)^{+}=\max (x, 0)$. If $k=k^{\prime}$, we have

$$
r_{k}\left(t_{1}+1\right)=r_{k}^{\prime}\left(t_{1}+1\right)=\left(d\left(t_{1}\right)-1\right)^{+} .
$$

If $k \neq k^{\prime}$, we have

$$
\begin{aligned}
r_{k^{\prime}}^{\prime}\left(t_{1}+1\right) & =\left(d\left(t_{1}\right)-1\right)^{+} \\
& \geq r_{k^{\prime}}^{\prime}\left(t_{1}\right) \\
& \geq r_{k^{\prime}}\left(t_{1}\right) \\
& \geq\left(r_{k^{\prime}}\left(t_{1}\right)-1\right)^{+} \\
& =r_{k^{\prime}}\left(t_{1}+1\right) \\
r_{k}^{\prime}\left(t_{1}+1\right) & =\left(r_{k}^{\prime}\left(t_{1}\right)-1\right)^{+} \\
& \geq\left(d\left(t_{1}\right)-1\right)^{+} \\
& =r_{k}\left(t_{1}+1\right) .
\end{aligned}
$$

From (32), (33), (34) and (35) we have

$$
\boldsymbol{r}\left(t_{1}+1\right) \leq \boldsymbol{r}^{\prime}\left(t_{1}+1\right) .
$$

From (30), (31), (36) and the induction hypothesis, it follows that (28) holds for $t_{2}-t_{1}=T+1$.

Now we prove Theorem 1.

Proof of Theorem 1. From Lemma 3 without loss of generality, we can assume that $\boldsymbol{r}\left(t_{1}\right)$ is a vector of decreasing entries. We prove this theorem by induction. If $t_{2}-t_{1}=0$,

$$
L_{\pi}\left(\boldsymbol{r}\left(t_{1}\right), t_{1}, t_{1}\right)=\ell_{\pi}\left(\boldsymbol{r}\left(t_{1}\right), t_{1}\right) .
$$

Since the sample path is feasible and policies $\pi$ and $\pi^{\star}$ are best effort policies, packets are lost only due to lack of NDLs. From (29), we have

$$
\ell_{\pi^{\star}}\left(\boldsymbol{r}\left(t_{1}\right), t_{1}\right)=\ell_{\pi}\left(\boldsymbol{r}\left(t_{1}\right), t_{1}\right) .
$$

Now we assume that (10) holds for any $t_{1}$ and $t_{2}$ such that $t_{2}-t_{1}=T$ and for any feasible sample path of the FDL.
We need to show that (10) holds for $t_{2}-t_{1}=T+1$ as well. Note that (31) holds for policy $\pi$ and $\pi^{\star}$. Now consider two systems identical to that in Figure 1. System I (resp. system II) assigns packets according to policy $\pi^{\star}$ (resp. policy $\pi)$. Assume that the arrival and departure instants of the two systems are identical and are generated by a feasible sample path of an FDL. Assume that the two systems have identical initial conditions. Denote the vectors of residual service times of the two systems by $\boldsymbol{r}^{I}(t)$ and $\boldsymbol{r}^{I I}(t)$ respectively. Since the initial conditions of the two systems are identical, we have

$$
\boldsymbol{r}^{I}\left(t_{1}\right)=\boldsymbol{r}^{I I}\left(t_{1}\right)=\boldsymbol{r}\left(t_{1}\right) \text {. }
$$

Let $k_{1}=\pi^{\star}\left(\boldsymbol{r}^{I}\left(t_{1}\right), d(t)\right)$ and $k_{2}=\pi\left(\boldsymbol{r}^{I I}\left(t_{1}\right), d(t)\right)$. Without loss of generality assume that $k_{1} \neq k_{2}$. Specifically, $k_{1}<k_{2}$ due to (7) and (8). Let $\tilde{\boldsymbol{r}}^{I I}\left(t_{1}+1\right)$ be a sorted version (in descending order) of vector $\boldsymbol{r}^{I I}\left(t_{1}+1\right)$. We now claim that

$$
\boldsymbol{r}^{I}\left(t_{1}+1\right) \leq \tilde{\boldsymbol{r}}^{I I}\left(t_{1}+1\right) .
$$

To prove this claim we first note that $\tilde{\boldsymbol{r}}^{I I}\left(t_{1}+1\right)$ is related to $\boldsymbol{r}^{I I}\left(t_{1}+1\right)$ by the following expression

$$
\tilde{r}_{j}^{I I}\left(t_{1}+1\right)= \begin{cases}r_{j}^{I I}\left(t_{1}+1\right), & \text { if } j<k_{1} \text { and } j>k_{2} ; \\ \left(d\left(t_{1}\right)-1\right)^{+}, & \text {if } j=k_{1} ; \\ r_{j-1}^{I I}\left(t_{1}+1\right), & \text { if } k_{1}<j \leq k_{2} .\end{cases}
$$

From (38), (46) and (40), it is easy to show that

$$
\tilde{r}_{j}^{I I}\left(t_{1}+1\right)= \begin{cases}\left(r_{j}\left(t_{1}\right)-1\right)^{+}, & \text {if } j<k_{1} \text { and } j>k_{2} ; \\ \left(d\left(t_{1}\right)-1\right)^{+}, & \text {if } j=k_{1} ; \\ \left(r_{j-1}\left(t_{1}\right)-1\right)^{+}, & \text {if } k_{1}<j \leq k_{2} .\end{cases}
$$

On the other hand,

$$
r_{j}^{I}\left(t_{1}+1\right)= \begin{cases}\left(r_{j}\left(t_{1}\right)-1\right)^{+}, & \text {if } j \neq k_{1} \\ \left(d\left(t_{1}\right)-1\right)^{+}, & \text {if } j=k_{1} .\end{cases}
$$

From (41) and (42), $\tilde{r}_{j}^{I I}\left(t_{1}+1\right)=r_{j}^{I}\left(t_{1}+1\right)$ for $j \leq k_{1}$ or $j>k_{2}$. For $k_{1}<j \leq k_{2}$,

$$
\begin{aligned}
& r_{j}^{I}\left(t_{1}+1\right)=\left(r_{j}\left(t_{1}\right)-1\right)^{+} \\
& \leq\left(r_{j-1}\left(t_{1}\right)-1\right)^{+}=\tilde{r}_{j}^{I I}\left(t_{1}+1\right) .
\end{aligned}
$$

The claim in (39) follows.

Finally, we have

$$
\begin{aligned}
& L_{\pi^{\star}}\left(\boldsymbol{r}^{I}\left(t_{1}\right), t_{1}, t_{2}\right) \\
& =\ell_{\pi^{\star}}\left(\boldsymbol{r}^{I}\left(t_{1}\right), t_{1}\right)+L_{\pi^{\star}}\left(\boldsymbol{r}^{I}\left(t_{1}+1\right), t_{1}+1, t_{2}\right) \\
& \leq \ell_{\pi^{\star}}\left(\boldsymbol{r}\left(t_{1}\right), t_{1}\right)+L_{\pi^{\star}}\left(\tilde{\boldsymbol{r}}^{I I}\left(t_{1}+1\right), t_{1}+1, t_{2}\right) \\
& =\ell_{\pi^{\star}}\left(\boldsymbol{r}\left(t_{1}\right), t_{1}\right)+L_{\pi^{\star}}\left(\boldsymbol{r}^{I I}\left(t_{1}+1\right), t_{1}+1, t_{2}\right) \\
& \leq \ell_{\pi}\left(\boldsymbol{r}\left(t_{1}\right), t_{1}\right)+L_{\pi}\left(\boldsymbol{r}^{I I}\left(t_{1}+1\right), t_{1}+1, t_{2}\right) \\
& =L_{\pi}\left(\boldsymbol{r}^{I I}\left(t_{1}\right), t_{1}, t_{2}\right) .
\end{aligned}
$$

where (43) follows from (39) and Lemma 4, (44) follows from (27) and (45) follows from (37) and the induction hypothesis. The proof is completed. 


\section{APPENDIX B}

In this appendix we present the proof of Theorem 2. The proof of Theorem 2 needs the following lemma. This lemma characterizes an important property of the $\pi^{\star}$ policy.

Lemma 5 If $r_{1}(t) \geq r_{2}(t) \geq \ldots \geq r_{B}(t)$, then policy $\pi^{\star}$ assures that $r_{1}(t+1) \geq r_{2}(t+1) \geq \ldots \geq r_{B}(t+1)$ for any delay request $d(t+1)$.

Proof of Lemma 5. From Eqs. (5) and (6), the residual service times satisfy the following recursion

$r_{i}(t+1)= \begin{cases}(d(t)-1)^{+}, & \text {if the new arrival joins NDL } i \\ \left(r_{i}(t)-1\right)^{+}, & \text {otherwise. }\end{cases}$

If $d(t)=0$, then $r_{i}(t+1)=\left(r_{i}(t)-1\right)^{+}$for all $i$ and the claim of the lemma obviously follows. Suppose that $d(t)>0$ and that the new arrival joins NDL $k$. From the definition of $\pi^{\star}$ in (7) and (8) and defining $r_{0}(t) \equiv 0$, we have

$$
r_{k-1}(t) \geq d(t) \geq r_{k}(t) \geq r_{k+1}(t)
$$

and

$$
\begin{aligned}
r_{k-1}(t+1) & \geq\left(r_{k-1}(t)-1\right)^{+} \\
& \geq(d(t)-1)^{+}=r_{k}(t+1) \\
& \geq\left(r_{k}(t)-1\right)^{+} \\
& \geq\left(r_{k+1}(t)-1\right)^{+} \\
& =r_{k+1}(t+1) .
\end{aligned}
$$

The claim of the lemma follows.

A direct consequence of Lemma 5 is the following corollary which can be proved by a simple induction argument. We omit the proof.

Corollary 6 If $r_{1}(0) \geq r_{2}(0) \geq \ldots \geq r_{B}(0)$, then policy $\pi^{\star}$ assures that $r_{1}(t) \geq r_{2}(t) \geq \ldots \geq r_{B}(t)$ for any $t$.

Now we prove Theorem 2. We show that at any time there cannot be more than $\lceil(d+1) / 2\rceil$ NDLs occupied. Since we have shown in Section II that policy $\pi^{\star}$ minimizes the discounted packet loss, it will also require the least number of NDLs to exactly emulate an FDL. Thus, it suffices to assume that the system operates under the policy $\pi^{\star}$.

We prove the theorem by contradiction. Let $k=\lceil(d+1) / 2\rceil$. Suppose that at time $t, k$ NDLs are occupied and an arrival occurs requesting delay $d^{\prime}$. Suppose that $d^{\prime}$ is less than the residual service times of all the occupied NDLs. Thus, the new arrival will be assigned to an idle NDL. Then $r_{i}(t)=0$ for all $i=k+1, k+2, \ldots, B$. According to Corollary 6 , $r_{i}(t) \geq r_{i+1}(t)$ for all $1 \leq i \leq B-1$. If $r_{i+1}(t)>0$, then $r_{i}(t)$ is strictly greater than $r_{i+1}(t)$. We identify $k+1$ packets, one from each occupied NDL, as follows. For NDL $k+1$, let packet $P_{k+1}$ be the newly arrived packet. Recursively we define the rest of the packets. Suppose that packets $P_{k+1}, P_{k}, \ldots, P_{i+1}$, where $1 \leq i \leq k$, have been defined. In NDL $i$, let $P_{i}$ be any one of the packets that arrive earlier than $P_{i+1}$ but have a departure time later than that of $P_{i+1}$. Such a packet must exist in NDL $i$. Just prior to the arrival time of packet $P_{i+1}$ denoted by $\tau^{a}\left(P_{i+1}\right)$, from the definition of $\pi^{\star}$ in (7) we have

$r_{i}\left(\tau^{a}\left(P_{i+1}\right)-\right)>\tau^{d}\left(P_{i+1}\right)-\tau^{a}\left(P_{i+1}\right)>r_{i+1}\left(\tau^{a}\left(P_{i+1}\right)-\right)$.

Rearranging terms in the above equation, we have

$$
\begin{aligned}
\tau^{a}\left(P_{i+1}\right)+r_{i}\left(\tau^{a}\left(P_{i+1}\right)-\right) & >\tau^{d}\left(P_{i+1}\right) \\
& >\tau^{a}\left(P_{i+1}\right)+r_{i+1}\left(\tau^{a}\left(P_{i+1}\right)-\right) .
\end{aligned}
$$

This equation implies that the last packet in the $i^{\text {th }}$ NDL will depart after packet $P_{i+1}$. Since it is present in the system at the arrival time of $P_{i+1}$, it is qualified to be selected as packet $P_{i}$. From the definition of packets $P_{1}, P_{2}, \ldots, P_{k+1}$, it follows that

$$
\tau^{a}\left(P_{k+1}\right)>\tau^{a}\left(P_{k}\right)>\ldots>\tau^{a}\left(P_{1}\right)
$$

and

$$
\tau^{d}\left(P_{k+1}\right)<\tau^{d}\left(P_{k}\right)<\ldots<\tau^{d}\left(P_{1}\right),
$$

where $\tau^{d}(P)$ is the departure time of packet $P$. Since arrivals and departures can only occur singly in each time slot and $\tau^{d}\left(P_{k+1}\right) \geq \tau^{a}\left(P_{k+1}\right)$, from (47) and (48)

$$
\begin{aligned}
\tau^{d}\left(P_{1}\right)-\tau^{a}\left(P_{1}\right) & \geq 2 \times k \\
& \geq d+1 .
\end{aligned}
$$

Since the maximum delay is $d$, we obviously have reached a contradiction.

\section{REFERENCES}

[1] I. Chlamtac, A. Fumagalli, L. G. Kazovsky, P. Melman, W. H. Nelson, P. Poggiolini, M. Cerisola, A. N. M. M. Choudhury, T. K. Fong, R. T. Hofmeister, C. L. Lu, A. Mekkittikul, D. J. M. Sabido IX, C. J. Suh and E. W. M. Wong, "Cord: contention resolution by delay lines," IEEE Journal on Selected Areas in Communications, vol. 14, pp. 1014-1029, 1996.

[2] R. L. Cruz and J.-T. Tsai, "COD: alternative architectures for high speed packet switching," IEEE/ACM Transactions on Networking, vol. 4, pp. 11-21, February 1996.

[3] D. K. Hunter, D. Cotter, R. B. Ahmad, D. Cornwell, T. H. Gilfedder, P. J. Legg, and I. Andonovic, " $2 \times 2$ buffered switch fabrics for traffic routing, merging and shaping in photonic cell networks," IEEE Journal of Lightwave Technology, vol. 15, pp. 86-101, January 1997.

[4] D. K. Hunter, W. D. Cornwell, T. H. Gilfedder, A. Franzen, and I. Andonovic, "SLOB: a switch with large optical buffers for packet switching," IEEE Journal of Lightwave Technology, vol. 16, pp. 17251736, October 1998.

[5] C.-S. Chang, Y.-T. Chen, Duan-Shin Lee, Jay Cheng, "Multistage Constructions of Linear Compressors, Non-overtaking Delay Lines, and Flexible Delay Lines," IEEE Infocom 2006, Barcelona, Spain.

[6] C.-C. Chou, C.-S. Chang, D.-S. Lee, J. Cheng, "A Necessary and Sufficient Condition for the Construction of 2-to-1 Optical FIFO Multiplexers by a Single Crossbar Switch and Fiber Delay Lines," IEEE Transactions on Information Theory, Vol. 52, No. 10, pp. 4519-4531, October 2006.

[7] Po-Kai Huang, C.-S. Chang, Jay Cheng, D.-S. Lee, "Recursive Constructions of Parallel FIFO and LIFO Queues with Switched Delay Lines," IEEE Trans. on Information Theory, Vol. 53, No. 5, p. 1778-1798, May 2007.

[8] S.-Y. R. Li, "Algebraic Switching Theory and Broadband Applications," Academic Press, 2001. 